\title{
Drug induced-hepatotoxicity with concurrent use of adalimumab and mesalamine for the treatment of crohn's disease
}

\begin{abstract}
Objective: To describe hepatotoxicity in a patient receiving combination therapy with Mesalamine and Adalimumab for the treatment of Crohn's disease.

Case summary: A 33-year old female with a history of Crohn's disease presented with complaints of upper abdominal pain and bilious vomiting. She had been receiving Adalimumab and Mesalamine for treatment of Crohn's disease. A liver panel revealed alkaline phosphatase (ALP): $188 \mathrm{IU} / \mathrm{L}$, aspartate aminotransferase (AST): 1,638 IU/L, alanine transaminase (ALT): $592 \mathrm{IU} / \mathrm{L}$, total bilirubin: $2.5 \mathrm{mg} / \mathrm{dL}$, and direct bilirubin: 1.6 $\mathrm{mg} / \mathrm{dL}$. Adalimumab and Mesalamine were discontinued and by day 6 liver panel improved to ALP: $154 \mathrm{IU} / \mathrm{L}$, AST: $57 \mathrm{IU} / \mathrm{L}$, ALT: $163 \mathrm{IU} / \mathrm{L}$, and total bilirubin: $0.8 \mathrm{mg} / \mathrm{dL}$. Upon discharge Adalimumab was restarted and Mesalamine was discontinued.

Discussion: Although cases of Adalimumab or Mesalamine hepatotoxicity occurring with monotherapy have been published, the reported incidence of hepatotoxicity is low. No previous cases of hepatotoxicity occurring with this combination therapy have been reported.

Conclusion: Upon discontinuation of Adalimumab and Mesalamine the patient's liver function improved, leading to the conclusion of drug-induced hepatotoxicity. This case should highlight the importance of routine liver function tests in patients receiving Adalimumab and Mesalamine combination therapy, along with early recognition and medication discontinuation.
\end{abstract}

Keywords: hepatotoxicity, inflammatory bowel disease, adverse drug reactions, druginduced disorders, adalimumab, mesalamine, toxicity
Volume 2 Issue 2 - 2015

\author{
Lindsay Hahn, Dusten Asmussen, Jessica \\ Benson \\ Belmont University College of Pharmacy, USA
}

Correspondence: Lindsay Hahn, Belmont University College of Pharmacy, Belmont BLVD Nashville, TN 372I2, USA, Tel 615460-81 19, Fax 615-460-6537, Email lindsay.hahn@belmont.edu

Received: January 30, 2015 | Published: April 6, 2015
Abbreviations: AST, aspartate aminotransferase; ALT, alanine transaminase; ALP, alkaline phosphatase; CD, crohn's disease; AntiTNF, anti-tumor necrosis factor; CMV, cytomegalovirus; ASMA, anti-smooth muscle antibodies; ANA, antinuclear antibodies; AntiLKM, anti-liver kidney microsomal antibodies

\section{Introduction}

Crohn's disease (CD) is a disorder characterized by transmura inflammation of the gastrointestinal tract, and it can involve any area of the gastrointestinal tract from mouth to the perianal area. Symptoms include chronic or nocturnal diarrhea, abdominal pain, weight loss, fever, and rectal bleeding. Treatment depends on disease location, severity, and associated complications. ${ }^{1}$ Mild to moderate $\mathrm{CD}$ is commonly treated with mesalamine; however, new evidence shows that this approach is less effective than corticosteroids or budesonide. ${ }^{1,2}$ For patients diagnosed with moderate to severe active disease, anti-tumor necrosis factor (anti-TNF) agents are used as an alternative in patients who have failed adequate therapy with a corticosteroids or immunosuppressive agent. ${ }^{1,3}$

Adalimumab (Humira ${ }^{\circledR}$ ) is a humanized monoclonal anti-TNF antibody which currently has seven FDA indications: Crohn's disease, rheumatoid arthritis (RA), ankylosoid spondylitis, psoriatic arthritis (PsA), juvenile idiopathic arthritis, ulcerative colitis (UC) and moderate to-severe plaque psoriasis. It exerts its effect through inhibition of TNF, resulting in down regulation of the immune- mediated inflammatory response associated with Crohn's disease. ${ }^{4}$ Mesalamine (Asacol HD®) is an aminosalicylate FDA-approved for the treatment of moderately active ulcerative colitis in adults, but also commonly used in Crohn's disease. Mesalamine, a sulfafree derivative of sulfasalazine, is thought to exert it's effect through inhibition of cyclooxygenase and lipoxygenase. ${ }^{5,6}$ The concurrent use of Adalimumab and Mesalamine for the treatment of Crohn's disease is a common occurrence in moderate to severe disease. ${ }^{1}$ Although these two medications have several well documented adverse reactions requiring monitoring or discontinuation, the reported incidence of hepatotoxicity is low. Drug related hepatotoxicity can be defined as injury to the liver that is associated with impaired liver function caused by exposure to a drug or another noninfectious agent. ${ }^{7}$ Reports of mesalamine hepatotoxicity are only $2 \%$ with Adalimumab exhibiting a 5\% incidence of elevated alkaline phosphatase (ALP) and $0.9 \%-3.5 \%$ incidence of elevated alanine transaminase (ALT). ${ }^{4,6}$ The exact mechanism by which Adalimumab and mesalamine exert these hepatic effects is unknown.

A literature search was performed in Medline, IPA, and IDIS using the terms Adalimumab, mesalamine, hepatotoxicity, elevated transaminases, elevated liver enzymes, and liver injury. The package inserts of Adalimumab and mesalamine were used to determine incidences for hepatic toxicity in clinical trials. Several case reports were found detailing hepatotoxicity with Adalimumab or mesalamine monotherapy. ${ }^{8-15}$ In addition to these case reports, review articles and original research articles were found detailing this adverse effect. ${ }^{16-20}$ 
However, no case reports were found describing hepatotoxicity with mesalamine and Adalimumab combination therapy. To the authors' knowledge this is first report detailing this occurrence.

\section{Case report}

A 33 year old white female weighing $77 \mathrm{~kg}$ with a history of Crohn's disease, diagnosed in 2012, and gastro esophageal reflux disease was hospitalized for severe upper abdominal pain associated with nausea and bilious vomiting. She described the pain as intermittent, with severe cramping. Her pain resolved with hydromorphone in the emergency department. She explained that the pain seemed to be worse in the morning, and that she had never experienced this type of pain during a Crohn's flare-up before. The patient was afebrile, and denied the use of acetaminophen, recreational drugs, or recent alcohol exposure. Upon physical exam, she had positive bowel sounds with no organomegaly, guarding, or rebound.

The patient's home drug regimen included mesalamine $1,600 \mathrm{mg}$ PO BID, Adalimumab 40mg subcutaneously every 2 weeks, dexlansoprazole $60 \mathrm{mg}$ PO daily, vitamin B12 injection monthly, vitamin B complex daily, and fexofenadine $180 \mathrm{mg}$ daily as needed. Her social history was negative for tobacco use, and she stated that she only consumed alcohol occasionally. Family history included a mother with diabetes, and a father who passed away from a myocardial infarction at the age of 71 . Her past surgical history was significant for cholecystectomy, and the patient had previously received the hepatitis B vaccination. The patient's blood alcohol level was undetectable, and the toxicology screen was negative. All vitals were within normal limits at the time of her visit. The patient's laboratory values were all within normal limits with the exception of her liver panel, which revealed the following results: ALP: $188 \mathrm{IU} / \mathrm{L}$, aspartate aminotransferase (AST): 1,638 IU/L, ALT: $592 \mathrm{IU} / \mathrm{L}$, total bilirubin: $2.5 \mathrm{mg} / \mathrm{dL}$, and direct bilirubin: $1.6 \mathrm{mg} / \mathrm{dL}$.

Upon admission, the patient's Adalimumab and mesalamine were discontinued. She was given supportive care for her pain symptoms, which resolved with the administration of hydromorphone. Gastroenterology was consulted, and hepatocellular injury was initially expected due to the extreme elevations in AST and only mildly elevated total bilirubin. The gastroenterologist acknowledged that this could be a result of drug therapy with mesalamine, Adalimumab, or both combined. A hepatitis panel was completed, and was negative for hepatitis A, B, and C. Ceruloplasmin was also ordered, to rule out Wilson's disease, and returned within normal limits. A magnetic resonance cholangipancreatography (MRCP) was performed, which indicated minimal nonspecific perihepatic free fluid and minimal hepatitis with no obstruction. A computed tomography (CT) scan was also completed revealing moderate mid-distal small bowel distention, air-fluid levels with mid wall thickening, and edema suspicious for ileus or partial obstruction. By day two of admission the patient's liver panel began to improve with ALP: 174 IU/L, AST: 696 IU/L, ALT: 701 $\mathrm{IU} / \mathrm{L}$, and total bilirubin: $3.3 \mathrm{mg} / \mathrm{dL}$. On day three of admission surgery was consulted and the patient eventually underwent an ileocecectomy with primary anastomosis. That same day the patient's liver panel continued to improve revealing the following laboratory values, ALP: $156 \mathrm{IU} / \mathrm{L}$, AST: $227 \mathrm{IU} / \mathrm{L}$, ALT: $411 \mathrm{IU} / \mathrm{L}$, and total bilirubin: $1.7 \mathrm{mg} /$ dL. Her hospital stay totaled 9 days, with her final liver panel on day 6 revealing ALP:154 IU/L, AST: $57 \mathrm{IU} / \mathrm{L}$, ALT: $163 \mathrm{IU} / \mathrm{L}$ and total bilirubin: $0.8 \mathrm{mg} / \mathrm{dL}$. She was discharged with instructions to resume all home medications, with the exception of Adalimumab. The patient was lost to follow up, and no further laboratory values are available.

\section{Discussion}

The characteristic presentation of hepatocellular or cytolytic injury involves elevated serum ALT, preceding an increase in total bilirubin and a modest increase in ALP. Drug related hepatotoxicity should be suspected when patients present with symptoms of anorexia, nausea, and fatigue. In addition to drug-related causes, other causes of liver dysfunction must be ruled out including hepatitis, alcoholic hepatitis, autoimmune hepatitis, biliary tract dysfunction, and hemodynamic disorders. $^{7}$ In our case, all possible causes of liver dysfunction were ruled out, with the exception of autoimmune hepatitis. Also, the patient's liver function tests (LFTs) returned to normal upon discontinuation of both mesalamine and Adalimumab. Utilization of the Naranjo probability scale indicated a probable probability that mesalamine and Adalimumab were associated with this case of hepatotoxicity. ${ }^{21}$

Several case reports were found detailing hepatotoxicity with Adalimumab or mesalamine monotherapy. ${ }^{8-15}$ One such case report details a patient treated with Adalimumab for rheumatoid arthritis. This patient was also suffering from hepatitis $\mathrm{C}$ but exhibited stable LFTs prior to Adalimumab initiation. Three months after treatment with Adalimumab, the patient showed an increase Gamma-glutamyl transpeptidase (GGT) to 23 times the upper limit of normal and ALP to 2.5 times the upper limit of normal. It is interesting to note that the patient showed no evidence of elevated AST or ALT. This pattern of LFT elevation is suggestive of damage to the liver or the gallbladder. Six months later Adalimumab was discontinued, and the patient's GGT and ALP elevations resolved. The authors concluded that the immediate resolution of these elevations upon discontinuation of Adalimumab is highly suggestive that Adalimumab was the causative agent. ${ }^{13}$ In a second case report, a patient had no previous history of liver disease or concomitant risk factors for liver disease and developed sub acute liver failure during therapy with Adalimumab for psoriatic arthritis. The patient was a 44 year old female with a 13 year history of psoriasis with a previous unsuccessful trial of methotrexate who was initiated on Adalimumab five months earlier. The treatment regimen consisted of $80 \mathrm{mg}$ Adalimumab subcutaneously at week 1, followed by $40 \mathrm{mg}$ every 2 weeks. She tested negative for tuberculosis and her LFTs were within the normal range before starting Adalimumab. Her symptoms of psoriasis resolved, but LFTs 4 months after initiation revealed an elevated ALT of $3.36 \mu \mathrm{mol} / \mathrm{L}$ (normal $<0.56 \mu \mathrm{mol} / \mathrm{L}$ ) and AST of $1.96 \mu \mathrm{mol} / \mathrm{L}$ (normal $<0.52 \mu \mathrm{mol} / \mathrm{L}$ ). Due to suspected hepatotoxicity from Adalimumab, the dosing interval was extended to $40 \mathrm{mg}$ every 3 weeks. Follow-up labs one month later, showed a significant increase of ALT $28.53 \mu \mathrm{mol} / \mathrm{L}$ and AST $14.35 \mu \mathrm{mol} / \mathrm{L}$, with an elevated total bilirubin of $60.6 \mu \mathrm{mol} / \mathrm{L}$ (normal $<21 \mu \mathrm{mol} / \mathrm{L}$ ). Adalimumab was immediately discontinued. Physical exam revealed an enlarged liver and jaundice. Liver biopsy indicated a sub acute hepatitis with perivenular necrosis of hepatocytes and cholestatic features, indicating acute toxic hepatitis. The patient tested negative for antibodies to hepatitis A, B, C, E and Cytomegalovirus (CMV) and had normal values of ceruloplasmin, ferritin, iron, titers for antismooth muscle antibodies (ASMA), antinuclear antibodies (ANA) and anti-liver-kidney microsomal antibodies (anti-LKM). The ALT and AST levels continued to rise along with an increase in INR to 1.3 and ammonium to $75 \mu \mathrm{mol} / \mathrm{L}$ (normal $<38 \mu \mathrm{mol} / \mathrm{L}$ ). Prednisolone $75 \mathrm{mg}$ daily was initiated and the liver enzymes slowly returned to normal. The steroid was tapered and her liver function tests were normalized by the 3 month follow-up. The quick resolution of hepatitis with a steroid and discontinuation of Adalimumab indicates this medication was most likely the causative agent. ${ }^{12}$ 
Mesalamine hepatotoxicity has also been reported in the literature. A brief case report in the American Journal of Gastroenterology details a 42 year old male with ulcerative colitis who was receiving mesalamine $2.4 \mathrm{~g}$ daily. Two weeks after initiation he began to experience fever, and at 6 weeks he was admitted for further workup. Upon admission his laboratory values revealed AST 97 IU/L, ALT $147 \mathrm{IU} / \mathrm{L}$, ALP $800 \mathrm{IU} / \mathrm{L}$, and GGT $663 \mathrm{IU} / \mathrm{L}$. A clinical diagnosis of mesalamine-induced fever and granulomatous hepatitis was made and the medication was discontinued. Three days later his fever resolved and his liver panel gradually improved. ${ }^{14} \mathrm{~A}$ second case report details a 65 year old male receiving mesalamine $3 \mathrm{~g}$ daily for the treatment of Crohn's disease. All liver function tests were normal prior to initiation of mesalamine, and five months later AST and ALT both rose to 9 and 13 times the upper limit of normal, respectively. These increases were attributed to simvastatin use, and simvastatin was discontinued. Eight months later AST and ALT increased to 10 and 12 times the upper limit of normal, respectively, with ALP and bilirubin levels within normal limits. Mesalamine use was discontinued and liver function tests returned to normal within 2 months..$^{15}$ The patient's presentation in our case is similar to many of the case reports found in the literature. The patient experienced an increase in total bilirubin, ALP, AST, and ALT, which all resolved upon discontinuation of melamine and Adalimumab. Both mesalamine and Adalimumab are reported to cause hepatotoxicity in numerous ways, as is evident from reviewing the above case reports. Despite the evidence that points to these medications as causative agents, there are limitations to this case. Perhaps the biggest limitation is that the patient was lost to followup. In order to conclusively determine if the cause was mesalamine, Adalimumab, or the combination therapy, a follow-up liver panel after reinitiating Adalimumab is necessary. A second limitation is that testing was not completed for autoimmune hepatitis. However given the fact that laboratory values improved upon discontinuation of the mediations autoimmune hepatitis alone likely did not cause these abnormalities. Despite these limitations, the resolution of her laboratory abnormalities following the discontinuation of mesalamine and Adalimumab strongly suggests that at least one, if not both of these medications contributed to her hepatic dysfunction.

\section{Conclusion}

This case describes probably mesalamine and Adalimumab induced hepatotoxicity. Although there is literature available detailing hepatic toxicity, the reported incidence remains low. Although the case does not determine if either of these medications alone or in combination caused the liver injury, the conclusion can be made that upon discontinuation the patient's liver function improved. The information in this case should highlight the importance of routine liver function tests in patients receiving mesalamine and Adalimumab along with early recognition and medication discontinuation.

\section{Acknowledgments}

None.

\section{Conflicts of interest}

The author declares there is no conflict of interest.

\section{References}

1. Lichtenstein GR, Hanauer SB, Sandborn WJ. Practice Parameters Committee of American College of Gastroenterology. Management of Crohn's disease in adults. Am J Gastroenterol. 2009;104(2):465-483.
2. Gisbert JP, Chaparro M, Gomollón F. Common misconceptions about 5 -aminosalicylates and thiopurines in inflammatory bowel disease. World J Gastroenterol. 2011;17(30):3467-3478.

3. Thomson AB, Gupta M, Freeman HJ. Use of the tumor necrosis factorblockers for Crohn's disease. World J Gastroenterol. 2012;18(35):48234854.

4. Adalimumab. In. Micromedex 2.0 online. Greenwood Village (CO): Thompson Reuters.

5. Mesalamine. In. Micromedex 2.0 online. Greenwood Village (CO): Thompson Reuters. [updated 5/12/014; accessed 5/14/2014].

6. Asacol [package insert]. Warner Chilcott Deutschland GmbH. D-64331 Weiterstadt, Germany; 2010.

7. Navarro VJ, Senior JR. Drug-related hepatotoxicity. $N$ Engl J Med. 2006;354 (7):731-739.

8. Ghabril M, Bonkovsky HL, Kum C, et al. Liver injury from tumor necrosis factor- $\alpha$ antagonists: analysis of thirty-four cases. Clin Gastroenterol Hepatol. 2013;11(5):558-564.

9. Kaiser T, Moessner J, Patel K, et al. Life threatening liver disease during treatment with monoclonal antibodies. BMJ. 2009;338:b508.

10. Adar T, Mizrahi M, Pappo O, et al. Adalimumab-induced autoimmune hepatitis. J Clin Gastroenterol. 2010;44(1):e20-e22.

11. Grasland A, Sterpu R, Boussoukaya S, et al. Autoimmune hepatitis induced by adalimumab with successful switch to abatacept. Eur J Clin Pharmacol. 2012;68(5):895-898.

12. Hagel S, Bruns T, Theis B, et al. Subacute liver failure induced by adalimumab. Int $J$ Clin Pharmacol Ther. 2011;49(1):38-40.

13. Frider B, Bruno A, Ponte M, et al. Drug induced liver injury caused by adalimumab: a case report and review of the bibliography. Case Reports Hepatol. 2013; Article ID 406901:1-3.

14. Braun M, Fraser GM, Kunin M,et al. Mesalamine-induced granulomatous hepatitis. Am J Gastroenterol. 1999;94(7):1973-1974.

15. Deltenre P, Berson A, Marcellin P, et al. Mesalamine (5-aminosalicylic acid) induced chronic hepatitis. Gut. 1999;44(6):886-888.

16. Aithal GP. Hepatotoxicity related to antirheumatic drugs. Nat Rev Rheumatol. 2011;7(3):139-150.

17. Efe C. Drug induced autoimmune hepatitis and TNF- $\alpha$ blocking agents: Is there a real relationship. Autoimmun Rev. 2013;12(3):337-339.

18. Khokhar OS, Lewis JH. Hepatotoxicity of agents used in the management of inflammatory bowel disease. Dig Dis. 2010;28(3):508-518.

19. Gladman DD, Mease PJ, Ritchlin CT, et al. Adalimumab for longterm treatment of psoriatic arthritis: forty-eight week data from the adalimumab effectiveness in psoriatic arthritis trial. Arthritis Rheum. 2007;56(2):476-488.

20. Sokolove J, Strand V, Greenberg JD, et al. Risk of elevated liver enzymes associated with TNF inhibitor utilization in patients with rheumatoid arthritis. Ann Rheum Dis. 2010;69(9):1612-1617.

21. Naranjo CA, Busto U, Sellers EM, et al. A method of estimating the probability of adverse drug reactions. Clin Pharmacol Ther. 1981;30(2):239-245. 